Article

\title{
Development of a Robust Canine Welfare Assessment Protocol for Use in Dog (Canis Familiaris) Catch-Neuter-Return (CNR) Programmes
}

\author{
Heather Bacon ${ }^{1, *(1)}$, Hayley Walters ${ }^{1}$, Vlad Vancia ${ }^{2}$, Louise Connelly ${ }^{1}$ and Natalie Waran ${ }^{1,3}$ \\ 1 Jeanne Marchig International Centre for Animal Welfare Education, Royal (Dick) School of Veterinary \\ Studies, University of Edinburgh, Edinburgh EH25 9RG, UK \\ 2 The Romanian Society for Anthrozoology, Cluj 400372, Romania \\ 3 Faculty of Education, Humanities and Health Sciences, Eastern Institute of Technology, 501 Gloucester St, \\ Taradale, Napier 4112, New Zealand \\ * Correspondence: Heather.Bacon@ed.ac.uk; Tel.: +44-1316-517-438
}

Received: 2 July 2019; Accepted: 12 August 2019; Published: 16 August 2019

check for updates

Simple Summary: This paper describes the development of a robust composite welfare assessment tool to evaluate the welfare of free-roaming dogs passing through surgical sterilisation or catch-neuter-return (CNR) programmes. Catch-Neuter-Return programmes are frequently employed by governments and animal welfare charities to control the rising population of free-roaming dogs. Due to the focus on dog population control, individual dog welfare may be compromised through the CNR process, which comprises necessary stressors and harms, including capture, transport, surgery, and social disruption. Using a combination of a modified Delphi analysis and a Hazard Identification and a Critical Control Point analysis, this project described potential hazards to dog welfare during the CNR process and identified critical points where dog welfare could be assessed, and mitigations put in place to improve dog welfare throughout the CNR process. This project resulted in the development of a composite dog welfare assessment tool that will allow the robust assessment of the welfare of free-roaming dogs in CNR programmes and allow CNR projects to benchmark and mitigate hazards to dog welfare.

\begin{abstract}
The aim of this study was to develop a welfare assessment tool based on objective, reliable and relevant measures to be applied to individual dogs as they underwent a Catch-Neuter-Return (CNR) programme. A modified Delphi method and Hazard Analysis and Critical Control Points (HACCP) approach was used to develop the composite canine welfare assessment protocol, comprising both animal-based and resource-based measures. This draft welfare assessment protocol was then trialed and refined in existing CNR programmes to identify key control points where individual dog welfare may be moderately or significantly compromised in the CNR process. The results show that animal-based welfare indicators, e.g., pain behaviours, which provide a more direct indication of an animal's welfare state, require training and skill to recognise, whilst resource-based indicators are simple to measure but act only as indirect measures of welfare. We concluded that whilst CNR projects can potentially improve the health and welfare of free-roaming dogs in the long-term, the risk of short-term welfare harms during the CNR process is high. Thus, it is essential for staff involved in dog population management programmes to assess the welfare state of dogs in CNR and take remedial action to safeguard individual dog welfare.
\end{abstract}

Keywords: animal welfare; assessment; canis familiaris; dog; catch-neuter-return; CNR; Trap-neuter-return 


\section{Introduction}

Catch Neuter Return (CNR) programmes are often advocated by governments and animal welfare groups as being an appropriate solution to the control of dog populations [1-4]. CNR is considered by animal welfare charities, academics and the World Organisation for Animal Health (OIE) to be an essential tool in the control of dog populations, zoonoses and human-dog conflicts [5-8]. The ubiquitous nature of CNR, its application by leading animal welfare organisations, and the poor welfare implications of alternative dog population control measures, all contribute to the perception of CNR as a positive welfare intervention. CNR-type programmes are more likely to be utilised in more developed countries [9], where one may expect standards of veterinary training and dog handling to be higher. However, the variety of techniques used in CNR projects combined with the focus on population control, may result in poor welfare for individual dogs within the programme $[10,11]$.

The Hazard Analysis and Critical Control Point (HACCP) approach is used in a variety of industries to identify key stages within a process where potential hazards to a process may occur [12-14]. These stages are referred to as Critical Control Points (CCPs). The identification of potential hazards and CCPs allows for the development of evaluation and mitigation at these key points. The HACCP approach has been widely employed in the food security process to identify and rectify possible hazards to food safety and has been adapted for welfare assessment in the livestock industry [14-17]. Hazards were refined through a modified Delphi process. The Delphi process is a consensus stakeholder analysis [18] and has been utilised previously to identify hazards to animal welfare across a wide range of species [18-21]. Previous Delphi analyses in dogs and other species have relied on consensus from as few as seven [21] to as many as 154 [18] experts. In this project, we employed the HACCP approach for the first time to identify hazards that may compromise canine welfare in CNR programmes.

\section{Materials and Methods}

This was a purely observational study and no animals were used or harmed in this study. Ethical review was given by the Royal (Dick) school of veterinary studies Survey overview group ethics committee. No ethical review number was assigned.

The HACCP process is well documented and the approach as applied to this project is summarised in Table 1.

Table 1. The Hazard Analysis and Critical Control Point (HACCP) process as applied to this study.

\begin{tabular}{|c|c|c|}
\hline & HACCP Steps & Project Application \\
\hline 1. & Assemble the HACCP Team & Animal welfare research team at the University of Edinburgh \\
\hline 2. & Describe Product & $\begin{array}{l}\text { A robust composite canine welfare assessment tool that is practical } \\
\text { for use within the CNR environment }\end{array}$ \\
\hline 3. & Identify Intended use & To assess individual dog welfare in surgical CNR programmes \\
\hline 4. & $\begin{array}{l}\text { Construct a flow diagram to identify all stages } \\
\text { of the catch-neuter-return (CNR) process at } \\
\text { which CCPs may arise }\end{array}$ & $\begin{array}{l}\text { Five stages of CNR identified (capture and transport, caging, } \\
\text { peri-operative, post-operative and release) where CCPs may arise }\end{array}$ \\
\hline 5. & $\begin{array}{l}\text { List all potential hazards, conduct hazard } \\
\text { analysis, consider control measures }\end{array}$ & Via a Delphi analysis using experienced stakeholders \\
\hline 6. & $\begin{array}{l}\text { On site confirmation of CNR stages flow } \\
\text { diagram and potential welfare hazards }\end{array}$ & $\begin{array}{l}\text { Visit to an existing CNR programme to confirm CNR process and } \\
\text { trial of top-ranking suggested indicators from Delphi analysis as } \\
\text { markers of poor welfare at an existing CNR programme }\end{array}$ \\
\hline 7. & Determine CCPs & $\begin{array}{l}\text { Refinement of welfare indicator list as per CCP algorithm. Ensure } \\
\text { that all CCPs can be reliably identified }\end{array}$ \\
\hline 8. & Establish critical limits for each CCP & $\begin{array}{l}\text { Present or absent as all CCPs identified have a moderate-severe } \\
\text { welfare impact on the individual, thus identification of any CCP is a } \\
\text { welfare problem }\end{array}$ \\
\hline
\end{tabular}


Table 1. Cont.

\begin{tabular}{lll}
\hline \multicolumn{2}{c}{ HACCP Steps } & \multicolumn{1}{c}{ Project Application } \\
\hline 9. & Establish corrective action & Guidance on improving welfare \\
\hline 10. & Establish verification procedures & Visit to a second CNR programme to validate selected indicators \\
\hline 11. & Establish documentation and record keeping & Guidance notes on implementation of the welfare audit \\
\hline
\end{tabular}

\subsection{Defining Project Outcomes and the CNR Process}

The research team was assembled and ethical approval was obtained via the Veterinary and Human Ethical Review Committees at the University of Edinburgh's Royal (Dick) School of veterinary studies. The product—a robust canine welfare audit—was described: the key features identified were that it needed to be practical, economical and reliable, and it should identify moderate to significant welfare hazards within the CNR process, recognising that milder welfare insults are inevitable during a CNR intervention comprising of capture, transport and surgery. A flow diagram considered all aspects of the CNR process and identified seven primary stages of CNR (Figure 1), five of these stages were considered to impact dog welfare directly and were thus considered in this project. The seven stages identified were 1 . Surveying and planning - the logistical process and demographic survey that should be completed prior to undertaking a CNR project. As this stage is non-invasive to dogs, it was not considered to directly impact dog welfare, although it is essential for the success of a CNR programme. 2. Capture and transport-the removal of free-roaming dogs from the street and their transportation to the clinical site. 3. Caging/kenneling - the confinement of dogs at the clinical site prior to surgical intervention. 4. Perioperative-the time frame spanning dogs being removed from their cage/kennelling area and undergoing sedation to them being returned to their cage/kennelling area post-surgery. 5. Post-operative-The period of time between dogs returning to their cage/kennelling area after surgery and being released. 6 . Release-The processes including handling, transport and return to the release site. 7. Monitoring and Evaluation-the process of data analysis, follow-up surveying and evaluation of impact. As this stage is non-invasive to dogs, it was not considered to directly impact dog welfare, although it is essential for the success of a CNR programme.

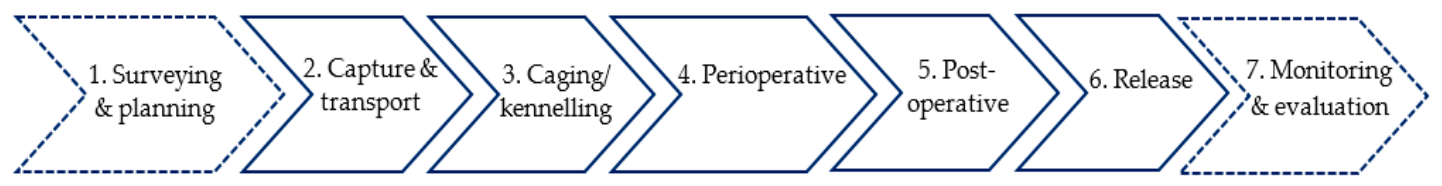

Figure 1. Flow diagram outlining the seven primary stages of $\mathrm{CNR}$. Solid outline $=$ stages that were determined to directly impact upon dog welfare.

\subsection{Hazard Identification}

Hazard identification was achieved through a sequential process of first reviewing the literature on existing dog welfare indicators, and secondly, conducting a two-stage modified Delphi process. A thorough review of the existing published and grey literature relating to dog population management, neutering, kenneling, veterinary care and canine welfare assessment was completed using Web of Science, Google scholar and PubMed, in addition to contacting relevant organisations working in the field for unpublished guidelines and protocols. This search identified behavioural, physiological and resource-based indicators that may be employed to identify hazards to canine welfare. A questionnaire of these canine welfare indicators developed from the literature review and from practical experiences within the research team was formulated. Each indicator on the list was assigned to one of the five stages of the CNR process based on the authors' experience and context from the literature. Some indicators were modified slightly to accommodate practical realities of CNR. For example, the anesthetic mortality in dogs is generally reported as $0.03-0.12 \%$ [22-24], however we initially set a threshold of mortality 
at $2 \%$, recognizing that pre-operative health assessment and prophylactic medical care was likely to be much more limited in street dogs and thus, the risks of unanticipated peri-operative deaths much higher. Due to the field trials, this threshold was later revised and lowered. Similarly, the threshold for surgical time was set at $90 \mathrm{~min}$, based on the durations reported in the literature at which infection risks significantly increased [25]. Some indicators were identified as being potentially useful in more than one stage.

\subsection{The Expert Panel}

A list of experienced stakeholders was developed by utilising a matrix approach to ensure that expertise across a breadth of dog management experience was sought. The categories of expertise utilised in the matrix were 'clinical CNR', 'dog behaviour, welfare, or pain assessment', 'dog population management', and 'dog kennelling and housing'. A maximum variation purposive sample was selected from veterinary organisations and non-governmental organisations (NGOs) working in the fields of canine medicine, CNR, or animal behaviour. A maximum purposive sample is a non-probability sampling technique that seeks to ensure a relevant sample of expertise is recruited. A minimum of five experts were recruited to each category of the matrix, resulting in 26 stakeholders. Experts were recruited based on their published work and experience in the field of CNR. Each expert was contacted confidentially with an introduction to the project and the option to decline involvement. Following this, one respondent withdrew from the process, leaving 25 participants.

\subsection{The Modified Delphi Process}

A two-stage process was employed. In the first stage of the Delphi analysis, experts were provided with the questionnaire, including the list of potential behavioural, physiological and resource-based welfare indicators for each stage of the CNR process and asked to contribute to the list with any further suggested indicators or hazards to dog welfare in CNR, based on their own experience.

The expert panel $(n=25)$ was then asked to score each indicator for practicality (how easily it could be utilised in a field CNR situation), economics (its value for money), and validity (how accurately it represented the dog's welfare state). Each indicator received 1 point when scored by the respondents for any of the criteria 'practical', 'economical', or valid' (maximum of 3 points per indicator per respondent).

The complete list of indicators was then re-sent to the 25 original Delphi participants in a second round where participants were asked to score each indicator/hazard according to the level of welfare compromise it represented on a scale of mild-moderate-severe. Mild was defined as 'short-term or minor physical or mental welfare compromise with which the dog can cope and adapt'. Moderate was defined as 'a welfare compromise which is likely to cause behavioural or mental distress or physical pain or injury, but which does not result in long-term effects'. Severe was defined as 'a level of welfare compromise which is likely to cause long-term physical discomfort/impairment or on-going mental/behavioural distress, or affect the dog's survivability'.

Each indicator/hazard received 1 point when scored by the Delphi respondents for either of the criteria 'moderate' or 'severe' welfare compromise (maximum of 1 point per indicator per respondent). Moderate and severe indicators received the same score, as we were simply interested in distinguishing them from 'mild' indicators, not attempting to rank these indicators in any way. Indicators scored as 'mild' welfare compromise received zero points during this round, as the research team considered that mild welfare compromise was inevitable during the handling and surgery of dogs, and by definition, a mild welfare insult was one that dogs would be able to cope with during the CNR process.

The indicators and hazards were ranked according to response scores. There is a lack of accepted guidance on the level of group agreement required in this process e.g., 51\% [21,26], 80\% [27]. Whaytt et al. devised the process of ranking Delphi responses by calculating the percentage of the maximum response score (\%MRS) for each indicator [18] and this approach was also used in this project. We calculated the \%MRS for each indicator across the categories of practicality, reliability, economy and 
welfare significance. \%MRS ranged from 2.2\% (microchipping) to 100\% (post-operative pain behaviour). As mentioned above, MRS $>50 \%$ is often selected as a threshold for group agreement, however, in this project, because all the indicators were derived either from the existing evidence in the literature or from expert consensus, they were considered to be valid indicators of moderate-severe welfare problems and a low threshold of $30 \%$ MRS was set with all indicators scoring over this, retained in the study in order to allow a maximum number of indicators to be trialed in the field.

\subsection{Inter-Observer and Intra-Observer Reliability Testing}

The assessment of some welfare indicators and hazards was very straightforward, e.g., 'dog offered drinking water prior to release' or 'dog provided with bedding in kennel' was directly observable and not subject to interpretation, but some of the indicators listed were potentially open to interpretation. In particular, the behavioural or human-dog-interaction indicators had the potential for subjectivity in assessment, e.g., dog shows signs of 'post-operative pain', or 'acceptable handling technique used' may be assessed differently by different observers. To ensure inter-observer reliability in the field tests, two researchers with extensive practical experience in dog behaviour, handling and clinical care, performed inter- and intra-observer reliability testing, as described in [28] to ensure that they could consistently recognise and evaluate practices in free-roaming dog capture and handling, and in dog behavioural responses.

\subsection{Field Trial A-On-Site Confirmation and Initial Evaluation of Indicators}

Once it was established that the welfare indicators/hazards to welfare could be reliably identified by the research team, field sites to trial the composite welfare assessment were identified. The primary characteristics of the two field trial sites are shown in Table 2, but the sites were anonymized as trial site A and trial site B.

Table 2. Comparison of the primary characteristics of two CNR projects used as field trial sites, highlighting the variations in the two projects.

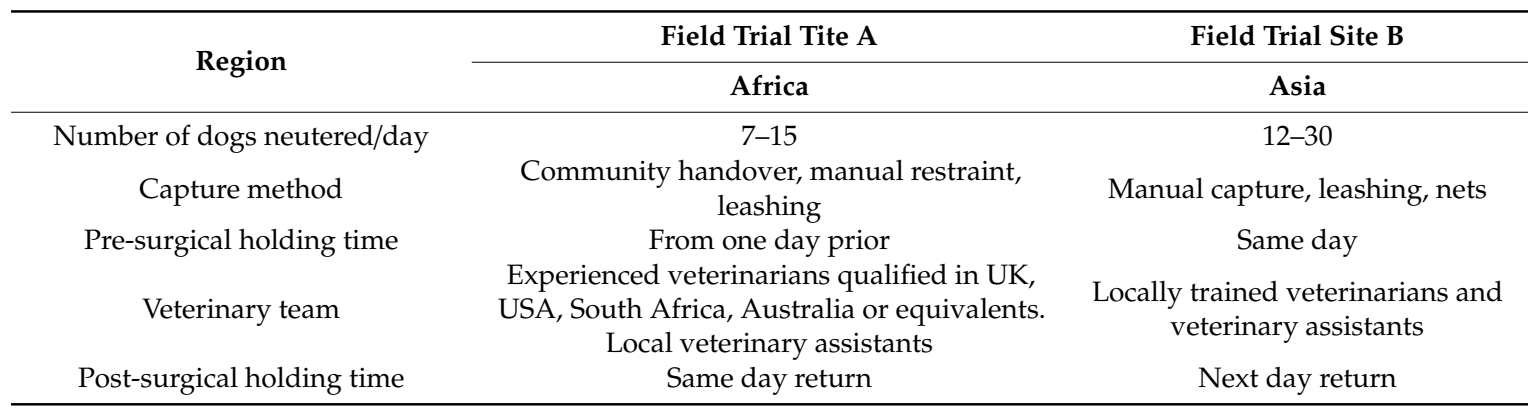

Field trial A consisted of a four-day test in which a convenience sample of 12 street dogs was followed throughout the entire process of a well-established CNR programme, with an additional 14 dogs observed for sections of the CNR process. Each of the 53 indicators on the draft audit was applied to the test dogs as they went through the CNR process.

Based on the trialing of the draft audit at the CNR programme, a number of suggested indicators were removed from the draft audit as they were found to be impractical, unreliable or not financially possible, e.g., heart rate variability, lymphocyte: neutrophil ratio.

Additionally, some indicators that had proven useful in more than one category were duplicated across categories where appropriate. One high-scoring indicator (pre-existing injury at time of capture $82 \%$ MRS) was removed from the study, as it was not thought to accurately reflect a welfare problem that was controllable within the scope of the CNR process, but instead reflected a pre-existing welfare problem. It also became apparent that some of the record-based and resource-based indicators, e.g., mortality rate, administration of vaccinations, and the presence of a crash kit, were either not related to a specific stage within the CNR process or not representative of individual dog welfare, but were 
instead programmatic indicators of good practice, therefore, a separate category was created on the draft audit for these indicators.

Based on this first field test, some of the descriptors of the CCPs were modified to minimise the potential for variation in interpretation by an observer. For example 'excessive force in capture' was changed to 'use of capture equipment, e.g., loop pole, net or neck grasper', as this equipment was consistently presented by Delphi survey respondents as a reason for unacceptable welfare in capture and handling, and the use of these tools is a more objective measurement than attempting to determine 'excessive' force. Similarly, 'use of excessive force in handling' (a negative welfare indicator) was modified to 'Handler checks dog's response before handling' (a positive indicator), as it was felt that 'excessive force' was too subjective a term and would create variation in assessment. If the handler checks a dog's response before handling by allowing it to sniff at the handler or by stroking it and gauging its response, this represents an understanding of tailoring animal handling techniques to an individual dog and would be a positive indicator.

A previous assessment of dog behaviour using video trials [28] had demonstrated that determining very specific welfare states, e.g., 'calm-alert' vs. 'calm-relaxed' was too difficult to be carried out reliably, and even determining 'positive' and 'negative' welfare states based on a dog's demeanour and behaviours generated variation in responses [28]. However, it was decided to retain some behavioural welfare indicators within the draft welfare audit, as animal-based indicators were recognised to be more robust than resource-based indicators $[18,19]$. These indicators included 'anxiety/fear behaviour', 'aggressive behaviour', 'escape behaviour', 'exploratory behaviour', and 'pain behaviour' and their retention was based on their high \%MRS and thus, expert assessment of their significance to dog welfare.

\subsection{Defining Critical Control Points (Ccps)}

The HACCP process relies on establishing Critical Control Points (CCPs) once all potential hazards are identified $[29,30]$. Each welfare indicator, representing a potential dog welfare hazard, generated through the HACCP process, was evaluated as a CCP, against a modified CCP algorithm (Figure 2) to determine its appropriateness as a critical control point. Based on this assessment, 46 reliable, economical and practical indicators of moderate to severe welfare compromise were included in the second draft dog welfare audit, and, as some indicators were duplicated across stages in the CNR process, e.g., escape behaviour, provision of water etc., this resulted in 66 potential CCPs or welfare evaluation points. The second draft of the dog welfare audit was piloted at a second field trial (field trial B). 


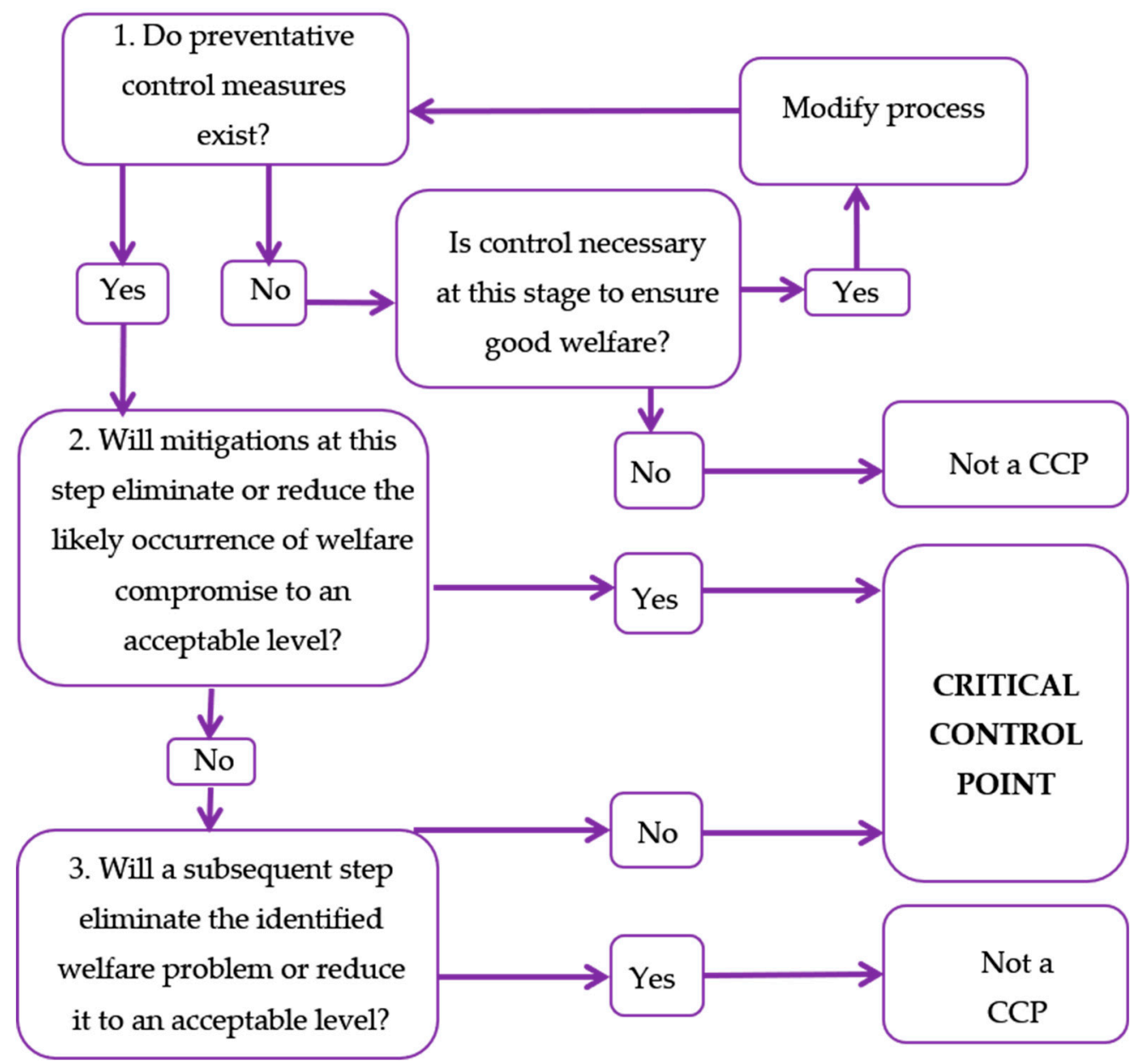

Figure 2. Critical control point algorithm (adapted from [31]).

\subsection{Field Trial B-Inter-Observer Reliability Testing}

The inter-observer reliability testing process was repeated with a three-observer team, including the two original observers who had already demonstrated consistency prior to field trial $\mathrm{B}$ as the behaviour-based CCPs included on the welfare audit had been edited and revised. It was important that despite these revisions, the behaviours they represented be consistently recognised by all three observers participating in field trial B. The results of a survey of CNR professionals in assessing dog behaviour had indicated that training in consistently identifying some or all of these behaviours is necessary even amongst experienced professionals working with dogs [28]. Each observer reviewed the behavioural indicators of emotional state as described by Tod et al. [32].

Inter-observer reliability was then tested between observers using ten dogs at a local shelter and veterinary hospital and a range of 28 video clips. Each observer viewed the behaviours shown by the observed dogs and individually recorded whether any of the five behavioural welfare indicators (aggression, escape, exploratory, fear, pain) included in the draft welfare audit and based on the descriptors published by Tod et al. was shown by each dog. Consensus in responses was evaluated using Fleiss' Kappa attribute agreement analysis in the Minitab 17 statistical software.

\subsection{Field Trial B-Verification of the Refined Welfare Audit}

The utility of the welfare audit was verified at a second CNR project field trial B in a different geographic location and with a different capture technique, veterinary capacity and return approach to ensure its flexibility across different programmatic structures. Three observers followed the dogs for five days, observing all stages of the CNR programme. Data were recorded using the welfare audit in 
Microsoft excel, Microsoft, Manchester, UK format on Psion Workabout Pro 3, Psion PLC, London, UK. In this field trial, a convenience sample of 53 dogs were followed from capture through to release or occasionally, euthanasia on humane grounds, and 88 additional dogs were evaluated during sections of the CNR process.

Following this final field trial, the CCPs on the draft audit were revised once more, for example programmatic indicators such as the $>2 \%$ mortality rate were discussed with the projects involved in the trials and the consensus was that this threshold was too lenient and thus it was lowered to $>1 \%$, still higher than the peri-operative mortality rate that might be anticipated in a more controlled surgical environment, but a realistic threshold for a CNR project dealing with dogs of unknown health status. Thus, the CNR dog welfare audit was finalised with expert input from the field trial projects. To enhance accessibility, this audit is available as a free mobile phone application (Dog Welfare Assessment app) on android and apple platforms [33].

\section{Results}

\subsection{Delphi Results}

We received 13 expert responses (52\% response rate) to round one of the modified Delphi process, resulting in 96 behavioural, physical, physiological and resource-based indicators of welfare or potential hazards to welfare. We received 11 expert responses (44\% response rate) to round two of the modified Delphi process. The ranking of the indicators above a 30\% MRS resulted in a list of 53 resource-based and animal-based (physical, physiological and behavioural) welfare indicators and hazards to be trialed as a draft audit (Appendix A).

\subsection{Field Trial A-Inter- and Intra-Observer Reliability Test}

It was essential that the research team be able to reliably recognise the animal-based indicators used in the welfare audit. Inter- and intra-observer reliability tests were evaluated for levels of agreement using a Fleiss Kappa test. These results are reported in [28].

\subsection{Field Trial B-Inter-Observer Reliability Test Results}

The second round of inter-observer reliability testing prior to Field Test B comprised three observers evaluating five behavioural CCPs using video footage and ten veterinary inpatient and shelter dogs. The test demonstrated agreement ranging from substantial to nearly perfect [34] between the three observers (Table 3).

Table 3. Results of the inter-attribute agreement analysis using Fleiss' Kappa, of inter-observer reliability trial undertaken prior to field trial B.

\begin{tabular}{ccccc}
\hline Attribute & Response & Inter-Observer K & $p$-Value & Agreement \\
\hline \multirow{4}{*}{ Demeanours } & Aggression & 0.845 & 0.00 & Nearly perfect \\
& Escape & 1.000 & 0.00 & Nearly perfect \\
& Exploratory & 1.000 & 0.00 & Nearly perfect \\
\multirow{2}{*}{ Pain } & Fear & 0.792 & 0.00 & Substantial \\
& Yes & 0.864 & 0.00 & Nearly perfect \\
& No & 0.864 & 0.00 & Nearly perfect \\
\hline
\end{tabular}

\subsection{The Final Canine Welfare Audit}

The final canine welfare audit is shown below (Table 4). It provides a mechanism for identifying gaps in care currently delivered to dogs going through a CNR programme and the quantification of the problems identified as a percentage of the total population of dogs. By using the welfare audit, individual CNR programmes may be systematically reviewed and critical control points currently not being considered may be identified in order to improve canine welfare within the programme. 
Table 4. Final welfare audit structure and valence of each CCP as a positive or negative welfare indicator.

\begin{tabular}{|c|c|c|}
\hline Critical Control Point & Definition & Valence \\
\hline \multicolumn{3}{|l|}{ Program } \\
\hline Provision of DHPPi vaccination & $\begin{array}{l}\text { Each dog entering the CNR programme receives DHPPi } \\
\text { vaccination }\end{array}$ & + \\
\hline Annual mortality rate $>1 \%$ & $\begin{array}{c}\text { Programme records are kept and the annual perioperative } \\
\text { mortality rate is recorded as }>1 \% \text { (excluding euthanasias on } \\
\text { humane grounds) }\end{array}$ & - \\
\hline $\begin{array}{l}\text { Facilities for treating additional } \\
\text { medical conditions }\end{array}$ & $\begin{array}{l}\text { Dogs presenting with additional medical or surgical needs } \\
\text { are appropriately treated or humanely euthanased }\end{array}$ & + \\
\hline Crash kit and drugs available & $\begin{array}{l}\text { Kit should include appropriate drugs and quick reference } \\
\text { dose charts in addition to CPR protocols }\end{array}$ & + \\
\hline Biosecurity/hygiene protocol in place & $\begin{array}{l}\text { A recognised process for cleaning all common areas should } \\
\text { be in place on at least a daily basis }\end{array}$ & + \\
\hline Positive community feedback on dogs & $\begin{array}{l}\text { Human residents demonstrate positive verbal and physical } \\
\text { interactions with dogs, and are supportive of the project }\end{array}$ & + \\
\hline \multicolumn{3}{|l|}{ Capture and Transport } \\
\hline $\begin{array}{l}\text { Presence of injury due to } \\
\text { capture/transport }\end{array}$ & $\begin{array}{l}\text { Dog shows signs of wounding or injury after } \\
\text { capture/transport }\end{array}$ & - \\
\hline Mortality at capture/transport & Dog is dead after capture/transport & - \\
\hline Use of capture equipment & $\begin{array}{l}\text { Neck graspers, catch poles, or nets are used to capture the } \\
\text { dog. }\end{array}$ & - \\
\hline Blood on equipment & $\begin{array}{l}\text { Blood on capture equipment indicating injury to at least one } \\
\qquad \operatorname{dog}\end{array}$ & - \\
\hline $\begin{array}{l}\text { Space to stand and lie comfortably in } \\
\text { transport }\end{array}$ & $\begin{array}{l}\text { Each dog is able to stand and lie down comfortably in the } \\
\text { transport vehicle }\end{array}$ & + \\
\hline Defecation/urination & Dog defecates or urinates on capture & - \\
\hline Escape behaviour & Dog runs away from handler & - \\
\hline Fear & $\begin{array}{l}\text { Ear tension: ears down, often tucked back against head, tail } \\
\text { tucked: tail tucked under hindlimbs, gaze aversion: won't } \\
\text { look directly at observer but turns or ducks head, whites of } \\
\text { eye: can see sclera around eye, front paw lift: raising of one } \\
\text { forepaw at a time }\end{array}$ & - \\
\hline Aggression to catcher/handler & $\begin{array}{l}\text { Baring of teeth, narrowing of eyes, raising of the hairs on the } \\
\text { neck and back, shifting of weight to allow escape, growling, } \\
\text { snarling and barking or snapping/biting directed towards } \\
\text { the human handler }\end{array}$ & - \\
\hline Inter-dog aggression & $\begin{array}{c}\text { Baring of teeth, narrowing of eyes, raising of the hairs on the } \\
\text { neck and back, shifting of weight to allow escape, growling, } \\
\text { snarling and barking or snapping/biting directed towards } \\
\text { another dog }\end{array}$ & - \\
\hline \multicolumn{3}{|l|}{ Cage/holding area indicators } \\
\hline Physical injury & $\begin{array}{c}\text { Dog shows signs of wounding or injury that were not } \\
\text { present upon capture }\end{array}$ & - \\
\hline Inter-dog aggression & $\begin{array}{c}\text { Baring of teeth, narrowing of eyes, raising of the hairs on the } \\
\text { neck and back, shifting of weight to allow escape, growling, } \\
\text { snarling and barking or snapping/biting directed towards } \\
\text { another dog }\end{array}$ & - \\
\hline Drinking water & $\begin{array}{c}\text { Easily accessible potable water is provided to the dog in } \\
\text { sufficient quantity }\end{array}$ & + \\
\hline Bedding material & $\begin{array}{l}\text { Dogs are provided with rubber matting, cardboard, } \\
\text { newspaper, fabric or similar absorbent material }\end{array}$ & + \\
\hline Signs of disease & $\begin{array}{l}\text { Dogs exhibit signs of infectious disease e.g., nasal or ocular } \\
\text { discharge, vomiting, pyrexia etc. }\end{array}$ & - \\
\hline Vocalisation & $\begin{array}{c}\text { Dog demonstrates repeated barking or howling behaviour } \\
\text { indicating distress at confinement }\end{array}$ & - \\
\hline
\end{tabular}


Table 4. Cont.

\begin{tabular}{|c|c|c|}
\hline Critical Control Point & Definition & Valence \\
\hline Escape behaviour & $\begin{array}{c}\text { Dog exhibits tunnelling, digging, wall bouncing, or biting } \\
\text { behaviour towards cage/enclosure barriers }\end{array}$ & - \\
\hline Fear & $\begin{array}{l}\text { Ear tension: ears down, often tucked back against head, tail } \\
\text { tucked: tail tucked under hindlimbs, gaze aversion: won't } \\
\text { look directly at observer but turns or ducks head, whites of } \\
\text { eye: can see sclera around eye, front paw lift: raising of one } \\
\text { forepaw at a time }\end{array}$ & - \\
\hline Aggression to handler & $\begin{array}{c}\text { Baring of teeth, narrowing of eyes, raising of the hairs on the } \\
\text { neck and back, shifting of weight to allow escape, growling, } \\
\text { snarling and barking or snapping/biting directed towards } \\
\text { the human handler }\end{array}$ & - \\
\hline \multicolumn{3}{|l|}{ Peri-operative } \\
\hline Handler tests dog & $\begin{array}{l}\text { Prior to handling dog, the human handler evaluates dog's } \\
\text { response to the human e.g., by slowly moving closer to dog, } \\
\text { crouching and offering a closed fist to sniff, or stroking the } \\
\qquad \text { dog }\end{array}$ & + \\
\hline Aggression shown to handler & $\begin{array}{l}\text { Baring of teeth, narrowing of eyes, raising of the hairs on the } \\
\text { neck and back, shifting of weight to allow escape, growling, } \\
\text { snarling and barking or snapping/biting directed towards } \\
\text { the human handler }\end{array}$ & - \\
\hline Dog injured during handling & $\begin{array}{c}\text { Dog shows signs of wounding or injury not present prior to } \\
\text { handling }\end{array}$ & - \\
\hline Analgesic drug administration & $\begin{array}{l}\text { Dog receives oral or parenteral administration of a } \\
\text { recognised analgesic e.g., a non-steroidal anti-inflammatory } \\
\text { drug, opioid, tramadol, and/or local anaesthestic infiltration. }\end{array}$ & + \\
\hline Peri-operative mortality & $\begin{array}{c}\text { Dog dies after anaesthesia is administered and prior to } \\
\text { recovery from anaesthesia }\end{array}$ & - \\
\hline Vocalisation during surgery & Dog emits audible noise during surgical procedure & - \\
\hline $\begin{array}{l}\text { Movement of head or forelimbs } \\
\text { during surgery }\end{array}$ & $\begin{array}{l}\text { Head, eyes, jaw or forelimbs exhibit spontaneous movement, } \\
\text { indicative of consciousness }\end{array}$ & - \\
\hline Break in aseptic technique & $\begin{array}{l}\text { Surgeon, surgical instruments or surgical area is } \\
\text { inappropriately prepared, or contaminated during the } \\
\text { surgical process }\end{array}$ & - \\
\hline Body condition score $1 / 3$ & $\begin{array}{l}\text { On a scale of } 1-3 \text { where } 1=\text { emaciation and } 3=\text { overweight, } \\
\text { the dog scores a } 1 \text {. Defined as "Bones easily visible (i.e., ribs, } \\
\text { pelvis, lumbar vertebrae); loss of muscle mass, obvious waist } \\
\text { and abdominal tuck" [29] }\end{array}$ & - \\
\hline $\begin{array}{l}\text { Surgery performed by student or } \\
\text { untrained vet }\end{array}$ & $\begin{array}{c}\text { Surgery performed by a non-qualified person e.g., a } \\
\text { veterinary student or a vet untrained in CNR surgical } \\
\text { procedures }\end{array}$ & - \\
\hline $\begin{array}{l}\text { Dedicated anaesthetic monitoring } \\
\text { person }\end{array}$ & $\begin{array}{l}\text { A person actively monitoring anaesthetic parameters e.g., } \\
\text { heart rate, respiratory rate, reflexes etc., and trained to } \\
\text { administer the correct dose of top-up anaesthesia under } \\
\text { veterinary direction in order to maintain a surgical plane of } \\
\text { anaesthesia }\end{array}$ & + \\
\hline Excessive surgical time & $\begin{array}{l}\text { Duration of surgery from initial incision to complete closure } \\
\qquad \text { of incision }>90 \mathrm{~min}\end{array}$ & - \\
\hline Ear tag placed & $\begin{array}{c}\text { Ear tag placed in pinna as post-operative identification of } \\
\text { neutering }\end{array}$ & - \\
\hline \multicolumn{3}{|l|}{ Post-operative } \\
\hline Drinking water & $\begin{array}{l}\text { Easily accessible potable water is provided to the dog in } \\
\text { sufficient quantity }\end{array}$ & + \\
\hline Post-operative analgesia & $\begin{array}{c}\text { Dog receives oral or parenteral administration of a } \\
\text { recognised analgesic e.g., a non-steroidal anti-inflammatory } \\
\text { drug, opioid, or tramadol. }\end{array}$ & + \\
\hline
\end{tabular}


Table 4. Cont.

\begin{tabular}{|c|c|c|}
\hline Critical Control Point & Definition & Valence \\
\hline Mortality & Dog dies after recovery from anaesthesia and prior to release & - \\
\hline Individual post-operative assessment & $\begin{array}{c}\text { Each dog is evaluated post-operatively for signs of pain, } \\
\text { infection or other problems }\end{array}$ & + \\
\hline Bedding material & $\begin{array}{l}\text { Dogs are provided with rubber matting, cardboard, } \\
\text { newspaper, fabric or similar absorbent material }\end{array}$ & + \\
\hline Poor quality recovery & $\begin{array}{l}\text { Dog exhibits staggering, disorientation, or a long } \\
\text { recovery period }\end{array}$ & - \\
\hline Pain behaviour & $\begin{array}{l}\text { Vocalising, looking at or interfering with incision, } \\
\text { hunched/tense posture, hunched/tense movement, } \\
\text { reluctance to move, facial tension/ears back/eyes squinting, } \\
\text { unresponsive/uninterested in interactions, nervous anxious } \\
\text { or fearful }\end{array}$ & - \\
\hline Escape behaviour & $\begin{array}{l}\text { Dog exhibits tunnelling, digging, wall bouncing, or biting } \\
\text { behaviour towards cage/enclosure barriers }\end{array}$ & - \\
\hline Fear & $\begin{array}{l}\text { Ear tension: ears down, often tucked back against head, tail } \\
\text { tucked: tail tucked under hindlimbs, gaze aversion: won't } \\
\text { look directly at observer but turns or ducks head, whites of } \\
\text { eye: can see sclera around eye, front paw lift: raising of one } \\
\text { forepaw at a time }\end{array}$ & - \\
\hline Aggression shown to handler & $\begin{array}{c}\text { Baring of teeth, narrowing of eyes, raising of the hairs on the } \\
\text { neck and back, shifting of weight to allow escape, growling, } \\
\text { snarling and barking or snapping/biting directed towards } \\
\text { the human handler }\end{array}$ & - \\
\hline Inter-dog aggression & $\begin{array}{l}\text { Baring of teeth, narrowing of eyes, raising of the hairs on the } \\
\text { neck and back, shifting of weight to allow escape, growling, } \\
\text { snarling and barking or snapping/biting directed towards } \\
\text { another dog }\end{array}$ & - \\
\hline Injury & $\begin{array}{c}\text { Dog shows signs of wounding or injury (except for } \\
\text { surgical incision) }\end{array}$ & - \\
\hline \multicolumn{3}{|l|}{ Release } \\
\hline $\begin{array}{l}\text { Released prior to gaining full motor } \\
\text { control or alertness }\end{array}$ & $\begin{array}{c}\text { Dog exhibits unsteady gait, drowsiness or disorientation } \\
\text { when released }\end{array}$ & - \\
\hline Individual assessment prior to release & $\begin{array}{l}\text { Dog is evaluated for pain, infection, hydration and level of } \\
\text { consciousness prior to release }\end{array}$ & + \\
\hline Reduced activity/physical impairment & $\begin{array}{l}\text { Dog exhibits reduced activity, reluctance to move, abnormal } \\
\text { gait or hunched or tense posture }\end{array}$ & - \\
\hline Released in a different location & $\begin{array}{c}\text { Dog is released in a location different from where it } \\
\text { was captured }\end{array}$ & - \\
\hline $\begin{array}{l}\text { Presence of post-operative } \\
\text { complication }\end{array}$ & $\begin{array}{l}\text { Surgical incision shows signs of swelling, redness discharge } \\
\text { or breakdown. Dog demonstrates an impairment related to } \\
\text { clinical treatment e.g., injection site pain or infection }\end{array}$ & - \\
\hline Released prior to food offered & $\begin{array}{l}\text { Dog is NOT offered palatable, appropriate food after } \\
\text { recovery from surgery and prior to release }\end{array}$ & - \\
\hline Released prior to water offered & $\begin{array}{l}\text { Easily accessible potable water is NOT provided to the dog } \\
\text { in sufficient quantity after recovery from surgery and prior } \\
\text { to release }\end{array}$ & - \\
\hline Use of capture equipment & $\begin{array}{c}\text { Neck graspers, catch poles, or nets are used to capture } \\
\text { the dog. }\end{array}$ & - \\
\hline Blood on equipment & $\begin{array}{l}\text { Blood on capture equipment indicating injury to at least } \\
\text { one dog }\end{array}$ & - \\
\hline Fear & $\begin{array}{l}\text { Ear tension: ears down, often tucked back against head, tail } \\
\text { tucked: tail tucked under hindlimbs, gaze aversion: won't } \\
\text { look directly at observer but turns or ducks head, whites of } \\
\text { eye: can see sclera around eye, front paw lift: raising of one } \\
\text { forepaw at a time }\end{array}$ & - \\
\hline
\end{tabular}


Table 4. Cont.

\begin{tabular}{|c|c|c|}
\hline Critical Control Point & Definition & Valence \\
\hline Aggression shown to handler & $\begin{array}{l}\text { Baring of teeth, narrowing of eyes, raising of the hairs on the } \\
\text { neck and back, shifting of weight to allow escape, growling, } \\
\text { snarling and barking or snapping/biting directed towards } \\
\text { the human handler }\end{array}$ & - \\
\hline \multirow[t]{2}{*}{ Inter-dog aggression } & $\begin{array}{c}\text { Baring of teeth, narrowing of eyes, raising of the hairs on the } \\
\text { neck and back, shifting of weight to allow escape, growling, } \\
\text { snarling and barking or snapping/biting directed towards } \\
\text { another dog }\end{array}$ & - \\
\hline & $\begin{array}{c}\text { Dog shows signs of wounding or injury (except for surgical } \\
\text { incision) }\end{array}$ & - \\
\hline
\end{tabular}

\subsection{Primary CCPs Detected}

Table 5 shows the most common problems detected at the two CNR projects participating in the study. The two projects are not directly comparable, as the indicators evaluated were refined and modified between the two field trials. However, the results do demonstrate the most common issues that arose within each of the two projects. The absence of a positively valenced CCP indicates a welfare concern and conversely, the presence of a negatively valenced CCP also indicates a welfare concern. The most common issue that arose in both projects was the recording of pain behaviours in either $56 \%$ (field site A) or $60 \%$ (field site B) of dogs, despite both projects administering a non-steroidal anti-inflammatory drug (NSAID) analgesic prior to surgery.

Table 5. Relative prevalence of selected CCPs as observed at the two CNR projects ' $\mathrm{A}$ ' and ' $\mathrm{B}$ '.

\begin{tabular}{cccc}
\hline Valence & Critical Control Point & CNR Project A (\%) & CNR Project B (\%) \\
\hline+ & Staff trained in dog capture & 0 & 100 \\
- & Vocalisation or movement of jaw or forelimbs & 22 & 17 \\
- & during surgery & 0 & 100 \\
- & Break in aseptic technique during surgery & 17 & 18 \\
- & Poor quality recovery & 56 & 60 \\
- & Post-operative pain behaviour & 43 & 0 \\
+ & Released prior to consumption of food/water & 0 & 17 \\
\hline
\end{tabular}

\section{Discussion}

This project applied the HACCP principles, a review of the literature, the modified Delphi method, and practical field trials to develop a robust, practical and economical canine welfare audit. The welfare audit proved to be a useful tool to assess dog welfare in the field. All three observers felt that it comprehensively evaluated all stages of CNR and flagged key areas of concern not previously identified by staff within the CNR programme. Different welfare problems were detected in CNR project $B$ when compared to CNR project $\mathrm{A}$, where many of the same indicators were used across both assessments, demonstrating the flexibility of the welfare audit in identifying a broad range of welfare problems across two CNR programmes with very different techniques.

Selecting the indicators used within the audit was a multiple-stage process founded in the literature and with expert input through the modified Delphi process. The literature was useful for generating many of the potential hazards/welfare indicators and for providing thresholds for surgical time and mortality rates [22,25]. This was a useful step as it generated potential indicators that had not been identified in the literature such as 'ear tag placed'. This example highlights the challenges of weighing up the cost-benefits within CNR programmes-all the dogs that pass through CNR programmes should be identified in order to prevent repeat capture at a later date and whilst the welfare implications of ear tagging may not be immediately obvious, it was suggested that ear tag placement could have longer-term detrimental impacts on welfare through the risks of chronic infection at the site of the tag 
or the risk of trauma should the tag be snagged. Alternative methods of marking neutered dogs such as ear-tipping were instead viewed as being less of a welfare risk.

Whilst acceptable and unacceptable limits are traditionally set for critical control points (CCPs), in order to maximise the practicality of the welfare audit in the field, a present/absent approach to CCPs was considered to be most appropriate for this assessment. For many of the CCPs, setting limits was not actually possible or practical, e.g., surgery was either performed by a student or an untrained vet or it was performed by an experienced vet, and drinking water was provided or it was not. In this study, any level of an identified welfare hazard would indicate an unacceptable deterioration in welfare state, as all suggested CCPs represented a moderate or severe welfare compromise, as ranked by the Delphi participants, therefore, scoring or scaling the various welfare hazards would have added an unnecessary complexity to the audit without necessarily providing any material benefit to dog welfare. As with any welfare assessment, this audit relies on proxy measures of an animal's welfare state if we accept that welfare is about how the animal feels [35], we also have to accept that any indirect measurement of these feelings will necessarily have the potential for error. The audit has attempted to incorporate multiple behaviours that may be rooted in similar negative affective states-for example, aggression and escape behaviour may well be rooted in anxiety/fear behaviour but by separating out these behavioural indicators we are maximizing the opportunity for these negative states to be recognized and for mitigations to be placed at the CCPs at which they occur.

In both field trials, the proportion of dogs observed showing signs of post-operative pain was similar, despite the administration of an analgesic drug in the peri-operative period. Factors influencing pain may include individual dog responses, surgical technique, tissue damage, and the post-operative environment [36-39]. Pain was recognised as a significant welfare problem but it can be minimised through the use of appropriate multimodal analgesia, such as the use of local anaesthesia alongside parenteral drugs, the employment of Halstead's principles of surgery by a competent veterinary surgeon, and adequate post-operative nursing care and ongoing analgesic provision [11,36,39]. However, these preventative and remedial steps can only be taken if the pain is first recognised. Additionally, other behavioural signs of negative welfare may be even more challenging to identify. Inter-observer reliability testing showed that the agreements for 'aggression', 'fear' and 'pain' were reduced when the observer team was increased from two to three observers for field trial B. Whilst the agreements remained at levels that were within the bounds of acceptability, these results highlight the challenges of consistently recognising behavioural indicators of poor welfare, even amongst individuals experienced in dog behaviour. One reason for this may be the confound between pain and fear behavior, which has been highlighted in free-roaming dogs [40], therefore, it is important that observers note pain-specific aspects of behaviour such as wound interference and orbital tightening, and not just general muscle tension when evaluating different emotional states. Further research is needed to establish specific pain indicators in free-roaming dogs.

Regardless of such challenges, animal-based indicators have been suggested as valid proxies for negative emotional states in dogs [32] and as such, we would recommend that rather than discarding these indicators as being difficult to interpret, resources be focused on training staff who handle and interact with dogs in recognizing these behavioural indicators. In situations where recognising behaviours indicating negative welfare states is challenging, efforts should be made to ensure that veterinary surgeons of adequate skill levels are employed and ongoing multimodal analgesics are provided.

A direct comparison between CNR projects may be unhelpful, as each project works under different circumstances and with access to different resources, however, the welfare audit enables CNR projects to 'benchmark' their own progress in improving dog welfare by giving them a means of recording the prevalence of CCPs. Repeating the audit at a later time enables a CNR project to compare its progress against its own previous audit scores and identify whether the remedial action against CCPs has been successful in positively impacting dog welfare. In order to facilitate this process, a 
free mobile phone 'Dog Welfare Assessment' app has been developed for both Apple and android platforms [33].

The audit also allows for funding bodies to identify 'good-practice' standards. For example, the lack of a person suitably experienced in anaesthetic monitoring meant that $17 \%-22 \%$ of dogs observed under anaesthesia displayed signs consistent with their plane of anaesthesia being unsuitable for surgery, e.g., spontaneous head movements, jaw movements, vocalisations, etc. By comparing similar numbers of dogs in projects with and without a person suitably experienced in anaesthetic monitoring, it would be possible to evaluate the impact of a person suitably experienced in anaesthetic monitoring on the proportion of dogs that display signs consistent with their plane of anaesthesia being unsuitable for surgery, and thus make recommendations as to the importance of such a person to dog welfare. This type of assessment might be helpful in determining priorities when funding bodies allocate resources to CNR projects.

Some distinct differences in welfare issues were highlighted between the two projects. For example, Project A focused heavily on surgical and veterinary standards, using only Royal college of veterinary surgeons (RCVS) or equivalently qualified vets with a minimum of two years veterinary experience, which resulted in good standards of surgical asepsis, but moderate or severe welfare hazards were identified in the dog capture and handling stages of the project where the staff were less well trained. Conversely, Project B had invested heavily in staff training on dog handling but employed locally trained vets who may have had insufficient practical skills [11], thus, the welfare issues identified there were clustered within the peri-operative period.

The field trials highlighted welfare problems within the programmes consistent with deficiencies in training highlighted in earlier research [28] —namely the recognition and management of pain and other behavioural indicators of negative welfare, as well as highlighting specific welfare hazards, such as a lack of aseptic technique, or the release of dogs prior to food/water consumption, which may be an issue within some programmes but not in others.

\section{Conclusions}

Catch Neuter Return programmes can provide a humane solution in dog population management, but it is important to recognise that the potential for dogs to experience negative welfare through the CNR process appears significant. Steps should be taken by CNR initiatives to ensure that staff are properly trained and resourced in order to ensure that they are able to recognise and mitigate negative dog welfare experiences. In particular, attention should be paid to measuring the welfare impacts on individual dogs experiencing CNR interventions, and not just on documenting the numbers of dogs neutered by a programme. This composite canine welfare audit is a practical, economical and robust tool, supporting CNR programmes in identifying and addressing potential welfare problems within their projects.

Author Contributions: Conceptualization, H.B., L.C. and N.W.; Data curation, H.B., H.W. and V.V.; Formal analysis, H.B.; Funding acquisition, H.B. and N.W.; Investigation, H.B. and H.W.; Methodology, H.B., H.W., V.V. and N.W.; Project administration, H.B., H.W., V.V., L.C. and N.W.; Resources, L.C.; Software, L.C.; Writing—original draft, H.B.; Writing—review \& editing, H.B.

Funding: This research was co-funded by Dogs Trust Worldwide.

Acknowledgments: The authors are grateful to the Dogs Trust canine welfare grant funding for their support of this project.

Conflicts of Interest: The authors declare no conflict of interest. 


\section{Appendix A}

Table A1. Table of 53 dog welfare indicators and hazards generated by the literature review and Delphi process with an MRS $>30$.

\begin{tabular}{|c|c|c|}
\hline & Capture Indicators & MRS\% \\
\hline 1 & Presence of injury due to capture & 95.6 \\
\hline 2 & Lack of trained staff & 32.6 \\
\hline 3 & $\begin{array}{c}\text { Extreme ambient temperature (respiratory alkalosis and hypocapnia at ambient } \\
\text { temperatures }>21^{\circ} \mathrm{C} \text {, elevated cortisol once core }>43 \mathrm{C} \text { ) }\end{array}$ & 65.2 \\
\hline 4 & Mortality & 65.2 \\
\hline 5 & Blood on equipment & 95.6 \\
\hline 6 & Barking/growling/aggression to handler & 78.3 \\
\hline 7 & Increasing Chase-Capture time & 76.1 \\
\hline 8 & Use of capture equipment e.g., nets, lasso (as opposed to manual handling) & 65.2 \\
\hline 9 & Escape behaviour & 60.1 \\
\hline 10 & Elevated respiratory rate & 43.5 \\
\hline 11 & Defecation/urination & 41.3 \\
\hline \multicolumn{3}{|c|}{ Kennelling/cage indicators } \\
\hline 12 & Physical injury & 84.8 \\
\hline 13 & Lack of biosecurity/hygiene protocol & 43.5 \\
\hline 14 & Inter-dog aggression & 30.4 \\
\hline 15 & Absence of drinking water & 84.8 \\
\hline 16 & Escape behaviour e.g., bar biting, tunnelling & 76.1 \\
\hline 17 & Absence of bedding material & 73.9 \\
\hline 18 & Vocalisation/barking/howling & 65.2 \\
\hline \multicolumn{3}{|c|}{ Peri-operative indicators } \\
\hline 19 & Absence of analgesic drug administration & 89.1 \\
\hline 20 & Aggressive restraint & 37.0 \\
\hline 21 & Peri-operative mortality & 37.0 \\
\hline 22 & Vocalisation/movement during surgery & 93.5 \\
\hline 23 & Break in aseptic technique & 65.2 \\
\hline 24 & Increased recovery time/poor quality recovery & 32.6 \\
\hline 25 & Emaciated at time of surgery & 30.4 \\
\hline 26 & Absence of crash kit & 30.4 \\
\hline 27 & Use of unqualified veterinary personnel or students for surgery & 30.4 \\
\hline 28 & Absence of dedicated anaesthetic monitoring person & 70.0 \\
\hline 29 & Aggression & 65.2 \\
\hline 30 & Decreased Heart rate variability (Increased sympathetic tone) & 65.2 \\
\hline 31 & Increasing surgical time & 65.2 \\
\hline 32 & Ear tag $(4 / 7)$ & 57.1 \\
\hline \multicolumn{3}{|c|}{ Post-operative indicators } \\
\hline 33 & Vocalisations (crying, whimpering, groaning, screaming). & 100.0 \\
\hline 34 & $\begin{array}{l}\text { Pain behaviours (hunching, abdominal guarding, restlessness, altered facial expression, } \\
\text { altered posture, increased body tension or flinching in response to gentle palpation of } \\
\text { injured area and palpation of regions likely to be painful, rigid) }\end{array}$ & 100.0 \\
\hline 35 & Absence of drinking water & 91.3 \\
\hline 36 & Absence of post-operative analgesia provision & 95.6 \\
\hline 37 & Attention to wound area (chewing, licking, looking, rubbing) & 89.1 \\
\hline 38 & Absence of post-operative assessment & 87.0 \\
\hline 39 & Absence of bedding material & 73.9 \\
\hline 40 & Noticeable behavioural change compared to pre-operatively & 84.8 \\
\hline 41 & Reduced exploratory and communicative behaviours & 76.1 \\
\hline 42 & Human avoidance/fear behaviour & 70.0 \\
\hline 43 & Escape behaviours & 63.0 \\
\hline 44 & Elevated/ decreased nasal planum temperature & 41.3 \\
\hline 45 & Mortality $>2 \%$ & 65.2 \\
\hline 46 & Lack of biosecurity & 30.4 \\
\hline 47 & Lack of staff to deliver care/monitoring & 30.4 \\
\hline
\end{tabular}


Table A1. Cont.

\begin{tabular}{lcc}
\hline & Release indicators & \\
\hline 48 & Released prior to regaining full motor control or alertness & 87.0 \\
49 & Reduced activity/physical impairment & 67.4 \\
50 & Released in different location & 39.1 \\
51 & Presence of post-op complications & 32.6 \\
52 & Released prior to consumption of food and water & 60.1 \\
53 & Released according to standard time schedule rather than individual assessment & 80.4 \\
\hline
\end{tabular}

\section{References}

1. International Companion Animal Management Coalition. Humane Dog Population Management Guidance. 2007. Available online: http://animal.coa.gov.tw/download/resources/d/09_resources_d0301.pdf (accessed on 26 June 2016).

2. Aidaros, H. Monitoring and control of dog populations. In Global Conference on Rabies Control: Towards Sustainable Prevention at the Source; OIE: Incheon, Korea, September 2011; pp. 7-9.

3. Kahn, S.; Stuardo, L.; Rahman, S.A. Oie guidelines on dog population control. Dev. Biol. 2008, 131, 511-516.

4. Jackman, J.; Rowan, A. Free-roaming dogs in developing countries: The benefits of capture, neuter, and return programs. In The State of the Animals; Salem, D.J., Rowan, A.N., Eds.; Humane Society Press: Washington, DC, USA, 2007; Volume 4, pp. 55-78.

5. ICAM. International Companion Animal Management Coalition. Available online: https://www.icamcoalition.org (accessed on 26 June 2016).

6. OIE. Putting the oie standards to work. In Proceedings of the 2nd OIE Global Conference on Animal Welfare, Cairo, Egypt, 22 October 2008.

7. FERA; WSPA; RSPCA; IFAW; HSI; OIE; WHO. 1st International Conference on Dog Population Management. Available online: http://www.stray-afp.org/blog/latest-news/1st-international-conference-ondog-population-management (accessed on 8 August 2019).

8. Reece, J.F. Dogs and dog control in developing countries. In The State of the Animals iii; Salem, D.J., Rowan, A.N., Eds.; Humane Society Press: Washington, DC, USA, 2005; pp. 55-64.

9. Dalla Villa, P.; Kahn, S.; Stuardo, L.; Iannetti, L.; Di Nardo, A.; Serpell, J.A. Free-roaming dog control among oie-member countries. Prev. Vet. Med. 2010, 97, 58-63. [CrossRef] [PubMed]

10. Warden, L. Simplicity, complexity and chaos in indian dog population management: What has gone wrong, what has gone right, what can be done and why should we persevere? In Proceedings of the Ist International Conference on Dog Population Management, York, UK, 4-8 September 2012.

11. Bacon, H.; Vancia, V.; Walters, H.; Waran, N. Canine trap-neuter-return: A critical review of potential welfare issues. Anim. Welf. 2017, 26, 281-292. [CrossRef]

12. Pierson, M.D.; Corlett, D.A. Haccp: Principles and Applications; Springer: New York, NY, USA, 1992; pp. $2-5$.

13. Mortimore, S.; Wallace, C. An introduction to haccp. In Haccp; Springer: New York, NY, USA, 1998; pp. 1-11.

14. Grandin, T. Effect of animal welfare audits of slaughter plants by a major fast food company on cattle handling and stunning practices. J. Am. Vet. Med. Assoc. 2000, 216, 848-851. [CrossRef] [PubMed]

15. von-Borell, E.; Bockisch, F.J.; Büscher, W.; Hoy, S.; Krieter, J.; Müller, C.; Parvizi, N.; Richter, T.; Rudovsky, A.; Sundrum, A.; et al. Critical control points for on-farm assessment of pig housing. Livest. Prod. Sci. 2001, 72, 177-184. [CrossRef]

16. Bell, N.J.; Bell, M.J.; Knowles, T.G.; Whay, H.R.; Main, D.J.; Webster, A.J.F. The development, implementation and testing of a lameness control programme based on haccp principles and designed for heifers on dairy farms. Vet. J. 2009, 180, 178-188. [CrossRef] [PubMed]

17. Edge, M.K.; Barnett, J.L. Development of animal welfare standards for the livestock transport industry: Process, challenges, and implementation. J. Vet. Behav. 2009, 4, 187-192. [CrossRef]

18. Whaytt, H.R.; Main, D.C.J.; Greent, L.E.; Webster, A.J.F. Animal-based measures for the assessment of welfare state of dairy cattle, pigs and laying hens: Consensus of expert opinion. Anim. Welf. 2003, 12, 205-217.

19. Webster, A.J.F.; Main, D.C.J.; Whay, H.R. Welfare assessment: Indices from clinical observation. Anim. Welf. 2004, 13, 93-98. 
20. Collins, J.; Hanlon, A.; More, S.J.; Wall, P.G.; Duggan, V. Policy delphi with vignette methodology as a tool to evaluate the perception of equine welfare. Vet. J. 2009, 181, 63-69. [CrossRef]

21. Buckland, E.L.; Corr, S.A.; Abeyesinghe, S.M.; Wathes, C.M. Prioritisation of companion dog welfare issues using expert consensus. Anim. Welf. 2014, 23, 39-46. [CrossRef]

22. Bille, C.; Auvigne, V.; Libermann, S.; Bomassi, E.; Durieux, P.; Rattez, E. Risk of anaesthetic mortality in dogs and cats: An observational cohort study of 3546 cases. Vet. Anaesth. Analg. 2012, 39, 59-68. [CrossRef] [PubMed]

23. Levy, J.K.; Bard, K.M.; Tucker, S.J.; Diskant, P.D.; Dingman, P.A. Perioperative mortality in cats and dogs undergoing spay or castration at a high-volume clinic. Vet. J. 2017, 224, 11-15. [CrossRef]

24. Matthews, N.S.; Mohn, T.J.; Yang, M.; Spofford, N.; Marsh, A.; Faunt, K.; Lund, E.M.; Lefebvre, S.L. Factors associated with anesthetic-related death in dogs and cats in primary care veterinary hospitals. J. Am. Vet. Med. Assoc. 2017, 250, 655-665. [CrossRef] [PubMed]

25. Vasseur, P.B.; Levy, J.; Dowd, E.; Eliot, J. Surgical wound infection rates in dogs and cats data from a teaching hospital. Vet. Surg. 1988, 17, 60-64. [CrossRef]

26. Loughlin, K.G.; Moore, L.F. Using delphi to achieve congruent objectives and activities in a pediatrics department. Acad. Med. 1979, 54, 101-106. [CrossRef]

27. Green, B.; Jones, M.; Hughes, D.; Williams, A. Applying the delphi technique in a study of gps' information requirements. Health Soc. Care Community 1999, 7, 198-205. [CrossRef]

28. Bacon, H.J.; Walters, H.; Vancia, V.; Waran, N. The recognition of canine pain behaviours, and potentially hazardous catch-neuter-return practices by animal care professionals. Anim. Welf. 2019, 28, 299-306.

29. Noordhuizen, J.P.T.M.; Metz, J.H.M. Quality control on dairy farms with emphasis on public health, food safety, animal health and welfare. Livest. Prod. Sci. 2005, 94, 51-59. [CrossRef]

30. Hulebak, K.L.; Schlosser, W. Hazard analysis and critical control point (haccp) history and conceptual overview. Risk Anal. 2002, 22, 547-552. [CrossRef]

31. Dewell, G. Pre-Harvest Food Safety. Available online: http://veterinarycalendar.dvm360.com/pre-harvestfood-safety-proceedings (accessed on 5 May 2019).

32. Tod, E.; Brander, D.; Waran, N. Efficacy of dog appeasing pheromone in reducing stress and fear related behaviour in shelter dogs. Appl. Anim. Behav. Sci. 2005, 93, 295-308. [CrossRef]

33. Jeanne Marchig International Centre for Animal Welfare Education. Dog Welfare Resources. 2018. Available online: http://animal.coa.gov.tw/download/resources/d/09_resources_d0301.pdf (accessed on 14 August 2019).

34. Landis, J.R.; Koch, G.G. The measurement of observer agreement for categorical data. Biometrics 1977, 33, 159-174. [CrossRef] [PubMed]

35. Duncan, I.J. Welfare is to do with what animals feel. J. Agric. Environ. Ethics 1993, 6, 8-14.

36. Hellyer, P.; Rodan, I.; Brunt, J.; Downing, R.; Hagedorn, J.E.; Robertson, S.A. Aaha/aafp pain management guidelines for dogs and cats. J. Feline. Med. Surg. 2007, 9, 466-480. [CrossRef] [PubMed]

37. Bednarski, R.; Grimm, K.; Harvey, R.; Lukasik, V.M.; Penn, W.S.; Sargent, B.; Spelts, K. Aaha anesthesia guidelines for dogs and cats. J. Am. Anim. Hosp. Assoc. 2011, 47, 377-385. [CrossRef]

38. Fossum, T.W. Small Animal Surgery Textbook; Elsevier Health Sciences: Oxford, UK, 2013; pp. $131-136$.

39. Goldberg, M.E. Analgesia for shelter medicine and trap-neuter-return. In Pain Management for Veterinary Technicians and Nurses; John Wiley \& Sons: Oxford, UK, 2014; Volume 147, pp. 147-156.

40. Greenaway, S.; Reece, J. Investigating Pain after Ovariohysterectomy (OHE) Surgery in Street Dogs. Available online: https://www.bva.co.uk/WorkArea/DownloadAsset.aspx?id=2147489876 (accessed on 13 June 2019).

(C) 2019 by the authors. Licensee MDPI, Basel, Switzerland. This article is an open access article distributed under the terms and conditions of the Creative Commons Attribution (CC BY) license (http://creativecommons.org/licenses/by/4.0/). 